\title{
Surface characterization of nitrogen-doped high purity niobium coupons compared with superconducting rf cavity performance
}

\author{
Pashupati Dhakalø and Gianluigi Ciovati® \\ Jefferson Lab, Newport News, Virginia 23606, USA \\ Uttar Pudasaini॰ \\ The College of William and Mary, Williamsburg, Virginia 23185, USA \\ Santosh Chetri®, Shreyas Balachandran $\odot$, and Peter J. Lee® \\ Applied Superconductivity Center, National High Magnetic Field Laboratory, \\ Tallahassee, Florida 32310, USA
}

(Received 13 September 2019; published 30 December 2019)

\begin{abstract}
We report the results from the measurements of high purity $\mathrm{Nb}$ samples and superconducting radiofrequency (SRF) cavities doped with nitrogen and followed by either electropolishing (EP) or buffered chemical polishing $(\mathrm{BCP})$, in order to understand the role of the postdoping treatment on the performance of SRF cavities. The samples characterization via scanning electron microscope, $\mathrm{x}$-ray photoelectron spectroscopy and secondary ion mass spectroscopy showed topographical differences on the surface of the samples after EP versus BCP treatment, but similar surface composition. Radio-frequency measurements were done on single cell cavities made from fine-grain and large-grain $\mathrm{Nb}$ treated by nitrogen doping followed by BCP and showed that improved $Q_{0}$ in the medium field in both fine-grain and large-grain cavities is possible with $\mathrm{BCP}$ postprocessing. However, there are differences between performances of large-grain versus fine-grain cavities after BCP. A cavity made from large-grain Nb showed a larger increase in $Q_{0}$ and a lower quench field compared to cavities made from fine-grain $\mathrm{Nb}$.
\end{abstract}

DOI: 10.1103/PhysRevAccelBeams.22.122002

\section{INTRODUCTION}

Advances in the processing of bulk superconducting radio frequency (SRF) niobium cavities in recent years via interior surface impurity diffusion of either titanium or nitrogen have resulted in significant improvements in their quality factor, $Q_{0}[1-4]$. The "nitrogen doping" technique anneals the niobium cavity at a temperature $\geq 800{ }^{\circ} \mathrm{C}$ in the presence of a partial pressure of nitrogen of $\sim 25 \mathrm{~m}$ Torr, which diffuses into the $\mathrm{Nb}$ surface. Normal conducting niobium nitride precipitates are also produced on the surface and must be subsequently removed using standard chemical methods, such as electropolishing (EP) or buffer chemical polishing (BCP). Recently, an alternative "nitrogen infusion" technique uses a lower temperature heat treatment $\left(120^{\circ} \mathrm{C}-200^{\circ} \mathrm{C}\right)$ for longer durations ( $\sim 8$ hours) in the presence of nitrogen and this also resulted in a higher quality factor without any degradation in accelerating gradient [5-7]. The benefit of the heat

Published by the American Physical Society under the terms of the Creative Commons Attribution 4.0 International license. Further distribution of this work must maintain attribution to the author(s) and the published article's title, journal citation, and DOI. treatment at a lower temperature over the high temperature nitrogen doping is the absence of postdoping electropolishing because no normal conducting precipitates are formed at the surface [7]. EP has been the conventional method to successfully remove the unwanted normal conducting niobium nitride from the surface of niobium cavities. Traditionally, BCP has also been used to treat cavity surfaces, however, there are no results reported on the use of BCP to remove the precipitate layers after the nitrogen doping process. One of the advantages that $\mathrm{BCP}$ has over EP is that the setup required for BCP is economical, and the process is less time intensive. In this study, we conduct a surface analysis of coupon samples that underwent replica SRF cavity treatments. Our driving questions are the following: (i) Are there differences in surface chemistry of samples after $\mathrm{N}$ doping followed by $\mathrm{BCP}$ versus EP? (ii) Can the high $Q_{0}$ advantage be maintained in N-doped cavity after BCP? (iii) How does $\mathrm{N}$ doping and subsequent $\mathrm{BCP}$ of a large-grain cavity compare to using EP?

Differences in surface structures and chemical composition are addressed by scanning electron, and atomic force microscopy (AFM), x-ray photoelectron spectroscopy (XPS), and time-of-flight secondary ion mass spectroscopy (TOF-SIMS). Cavity results on N-doped, and 
$\mathrm{BCP} / \mathrm{EP}$ 'ed samples are compared to investigate the key differences in performance.

\section{SAMPLE AND CAVITY PREPARATION}

Coupon samples $\left(10 \times 10 \times 2.8 \mathrm{~mm}^{3}\right.$ in dimensions $)$ labeled L-04 and L-06 were cut by wire electro-discharge machining from a high purity ( $R R R \sim 300)$ fine-grain (50 $\mu \mathrm{m}$ average grain size) niobium sheet. The samples were surface treated using BCP 1:1:1, removing $\sim 70 \mu \mathrm{m}$. Afterward, the samples were nanopolished at Cabot Microelectronics, USA, to obtain a surface with mirror quality smoothness. The samples were heat treated at $800^{\circ} \mathrm{C} / 3 \mathrm{hrs}$ followed by 2 minutes of exposure to nitrogen at pressure of $\sim 25$ mTorr. The nitrogen is then evacuated and the cavities were further annealed at $800^{\circ} \mathrm{C}$ for 6 minutes (2N6 recipe). The treatment of these samples attempted to replicate the cavity treatments as much as possible, most importantly the samples were heat treated inside the beam tube of $\mathrm{Nb}$ cavities.

$\mathrm{BCP}$ and EP have been the standard way of material removal from the SRF Nb surface after fabrication to remove the damaged layer and expose the fresh $\mathrm{Nb}$ surface, beneficial for the SRF performance. BCP uses a mixture of three acids: hydrofluoric acid (HF, 49\%), nitric acid $\left(\mathrm{HNO}_{3}, 69.5 \%\right)$ and phosphoric acid $\left(\mathrm{H}_{3} \mathrm{PO}_{4}, 85 \%\right)$. The typical proportion of the mixture used is $1: 1: 1$ or $1: 1: 2$ of ( $\mathrm{HF}: \mathrm{HNO}_{3}: \mathrm{H}_{3} \mathrm{PO}_{4}$ ). $\mathrm{HNO}_{3}$ reacts with $\mathrm{Nb}$ to form $\mathrm{Nb}_{2} \mathrm{O}_{5}$ which reacts with $\mathrm{HF}$ to form $\mathrm{NbF}_{5}$. The compound $\mathrm{NbF}_{5}$ is soluble in the solution and the repetition of the process removes the surface layers from the $\mathrm{Nb}$. The addition of $\mathrm{H}_{3} \mathrm{PO}_{4}$ is to slow down the process, "buffering" the exothermic process which would otherwise produce excessive heating. The standard EP acid mixture contains nine volume parts of sulphuric acid $\mathrm{H}_{2} \mathrm{SO}_{4}(96 \%)$ and 1 part of hydrofluoric acid $\mathrm{HF}(48 \%)$. EP is a surface finishing process whereby the anodization of $\mathrm{Nb}$ by $\mathrm{H}_{2} \mathrm{SO}_{4}$ forces the growth of $\mathrm{Nb}_{2} \mathrm{O}_{5}$ and $\mathrm{F}^{-}$dissolves $\mathrm{Nb}_{2} \mathrm{O}_{5}$ [8]. The setup for the EP process is more complex and the removal rate is slower compared to $\mathrm{BCP}$.

The sample L-04 was subjected to $\mathrm{BCP}$ with $\mathrm{HF}: \mathrm{HNO}_{3}: \mathrm{H}_{3} \mathrm{PO}_{4}=1: 1: 2$ by volume mixture to remove $\sim 6 \mu \mathrm{m}$ while keeping acid temperature below $12^{\circ} \mathrm{C}$ with etch rate $\sim 1 \mu \mathrm{m} / \mathrm{min}$, whereas sample L-06 was subjected to $\mathrm{EP}$ with $\mathrm{HF}: \mathrm{H}_{2} \mathrm{SO}_{4}=1: 9$ by volume at $\sim 30 \mathrm{C}$ with etch rate $0.5 \mu \mathrm{m} / \mathrm{min}$ to remove $\sim 6 \mu \mathrm{m}$ from sample, respectively [9]. Two single cell TESLA shaped $1.3 \mathrm{GHz}$ cavities $\left(B_{p} / E_{\text {acc }}=4.2 \mathrm{mT} /(\mathrm{MV} / \mathrm{m})\right) \quad[10]$ named RDTTD-01 and TD-05 were separately prepared with baseline measurement being $\sim 30 \mu \mathrm{m}$ EP and the same nitrogen doping protocol [11] was used before the cavities were subjected to BCP $1: 1: 1$ for inner surface removal. To precisely characterize the material removal from $\mathrm{BCP}$, a fresh batch of acid was prepared and the etch rate was calibrated on a flat $\mathrm{Nb}$ sample. Cavity RDTTD-01 was fabricated from high purity fine-grain $\mathrm{Nb}$ and $\mathrm{TD}-05$ was fabricated using high purity large-grain $\mathrm{Nb}$ from Tokyo Denkai.

Microscopy was performed in a Zeiss 1540 EsB field emission scanning electron microscope (FESEM) with a spatial resolution of $\sim 1 \mathrm{~nm}$. For the XPS study, the photoelectrons were excited using the x-ray source that produces the $\mathrm{MgK} \alpha$ radiation at $1253.6 \mathrm{eV}$ using a PHI 5100 system. Elemental spectra were acquired at various binding energy (B.E.) ranges in increments of $0.1 \mathrm{eV} /$ step using the electron energy analyzer operated in a constant energy mode with pass energy $71.55 \mathrm{eV}$. The data was averaged among ten cycles. Before acquiring the spectrum, the surface was lightly sputtered using $1 \mu \mathrm{A}$ argon ion at $1 \mathrm{keV}$ for 2 minutes to remove any hydrocarbons present on the surface. To characterize a range of sample depths, the XPS spectra were acquired at different takeoff angles $15^{\circ}$, $30^{\circ}, 45^{\circ}, 60^{\circ}$ and $75^{\circ}$ (at higher takeoff angles, signals are obtained from greater depths).

\section{COUPON CHARACTERIZATION}

\section{A. Surface morphology}

The FESEM secondary electron (SE) images of the surface of the coupon samples after the high-temperature nitrogen treatment are shown in Figs. 1(a) and 1(b). The entire surface is covered in small in-grain structures. These structures have been identified as nonsuperconducting niobium nitrides $\left(\mathrm{Nb}_{2} \mathrm{~N}\right)$ [12]. The formation of these nitride phases degrades the performance of SRF $\mathrm{Nb}$ cavities, so postdoping surface removal is required.

Figure 2 compares SE images of the surface of the $\mathrm{Nb}$ coupons after $\sim 6 \mu \mathrm{m}$ surface removal with (a) BCP and (b) EP. The SE images qualitatively show the differences in the surface roughness with these polishing techniques: The $\mathrm{BCP}$ treated $\mathrm{Nb}$ coupon has higher surface roughness compared to the EP treated $\mathrm{Nb}$ coupon. Ledges were produced on the surface of the $\mathrm{BCP}$ sample that are consistent with grain boundary grooving, and the difference in etching rates based on orientation in BCP treated $\mathrm{Nb}$. In contrast, the EP process does not etch the grain boundaries and different grains at different rates, leading to less roughness [13]. The backscattered electron (BSE) intensity in the images in Figs. 2(c) and 2(d) are sensitive to crystallographic orientation and show in-grain textural contrast for both the polycrystalline $\mathrm{Nb}$ coupons after BCP and EP respectively. Similar surfaces were observed for fine-grain high purity $\mathrm{Nb}$ in a low-temperature nitrogen exposure study [7]. These BSE images do not show any differences between $\mathrm{BCP}$ and $\mathrm{EP}$ on the $\mathrm{Nb}$ surface. The chemically polished $\mathrm{Nb}$ coupons were then cooled to $100 \mathrm{~K}$, held at $100 \mathrm{~K}$ for 30 minutes and warmed back up to room temperature. This procedure produces surface $\mathrm{Nb}$ hydrides during the cooling down cycle, in the temperature range of 70-140 K [14] in the presence of excess high 

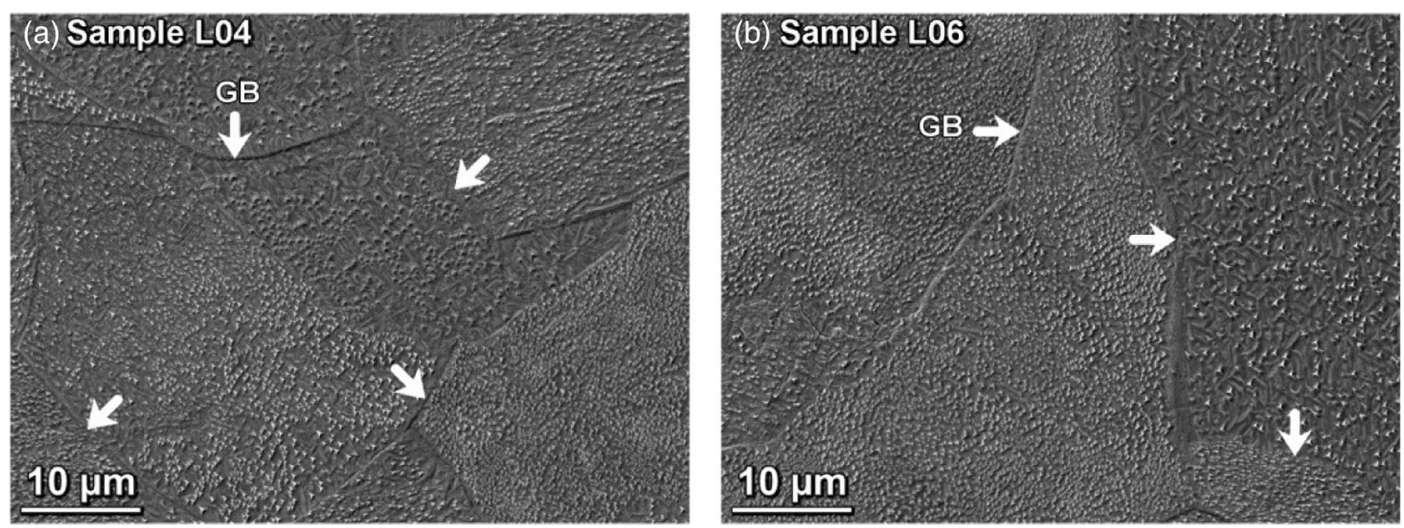

FIG. 1. FESEM SE images, showing the surface of two Nb coupons (a) L04 and (b) L06 both treated with high temperature nitrogen doping steps $\left(800^{\circ} \mathrm{C}\right.$ for 3 hours followed by nitrogen exposure at $800{ }^{\circ} \mathrm{C}$ for 2 minutes and anneal for 6 minutes without nitrogen). The surface shows the formation of nonsuperconducting niobium nitride phase.
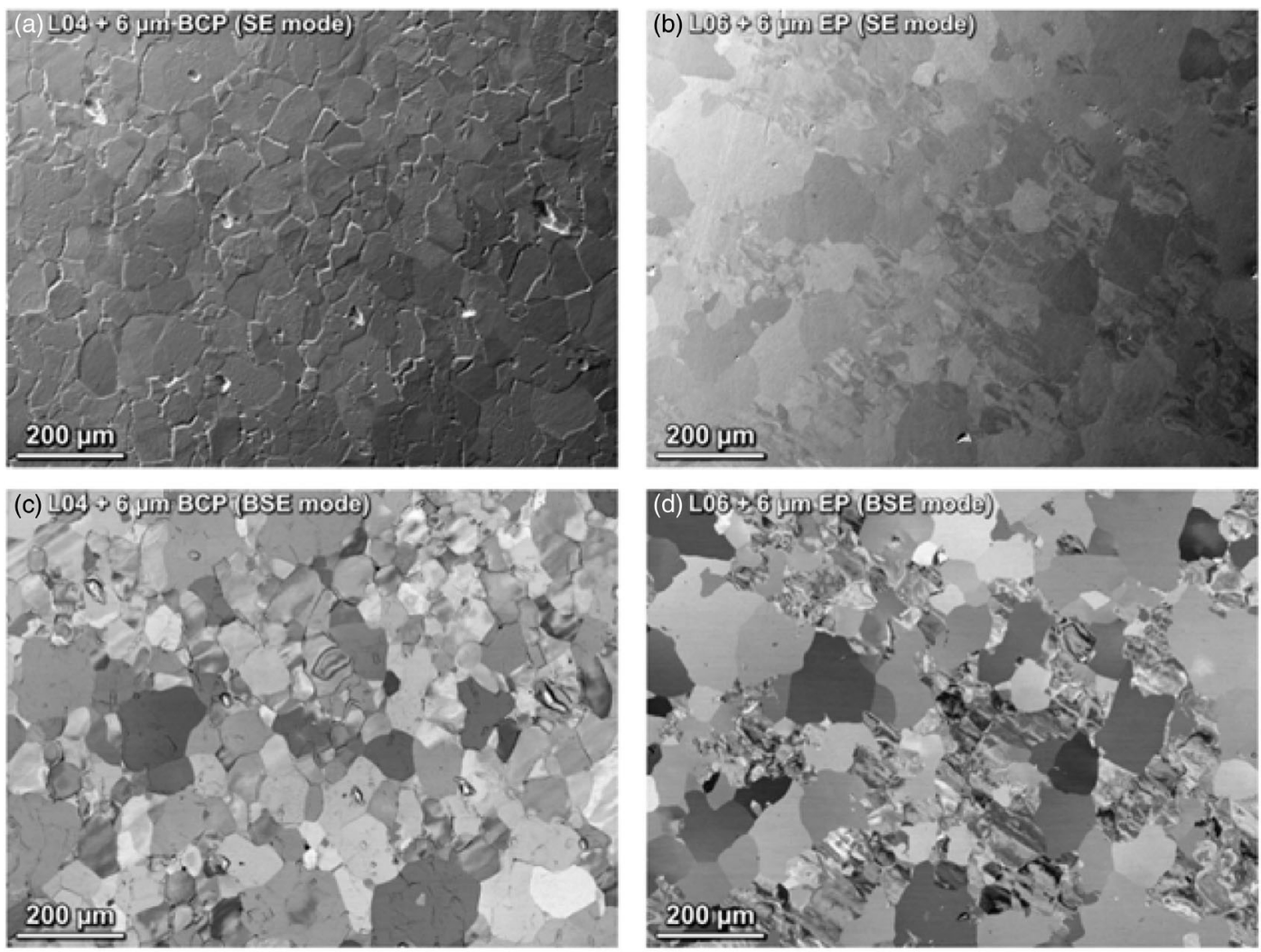

FIG. 2. (a) and (b) are SE mode SEM images of the surface after about $\sim 6 \mu \mathrm{m}$ surface removal from sample L04 with BCP and sample L06 with EP, (c) and (d) are their respective orientation-sensitive BSE mode images. SE mode image shows higher surface roughness on BCP treated surface.

surface hydrogen concentration in non-nitrogen treated samples. After this test for hydride formation we found no evidence by SEM imaging for strain scars left by hydrides on the surface of any of the samples [15].

Higher magnification SEM images of the surface with SEM imaging are shown in Fig. 3. The BCP surface shows densely packed structures, possibly due to the uneven etching of the $\mathrm{Nb}_{2} \mathrm{~N}$ and $\mathrm{Nb}$ on the $\mathrm{N}_{2}$ doped surface. The sample with EP shows no such features.

To further quantify the surface roughness, measurements by an atomic force microscope (AFM) were performed and the results are shown in Fig. 4. The rms surface roughness 

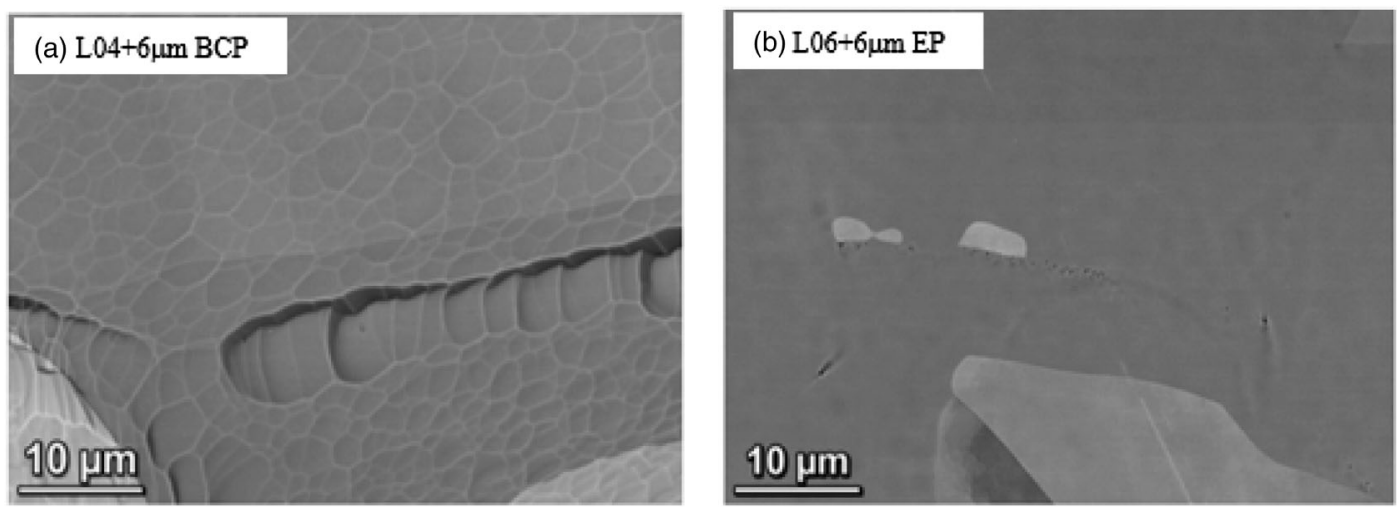

FIG. 3. Higher magnification SE mode SEM images of the coupon surfaces after about $~ 6 \mu$ m surface removal of (a) sample L04 with BCP and (b) sample L06 with EP.
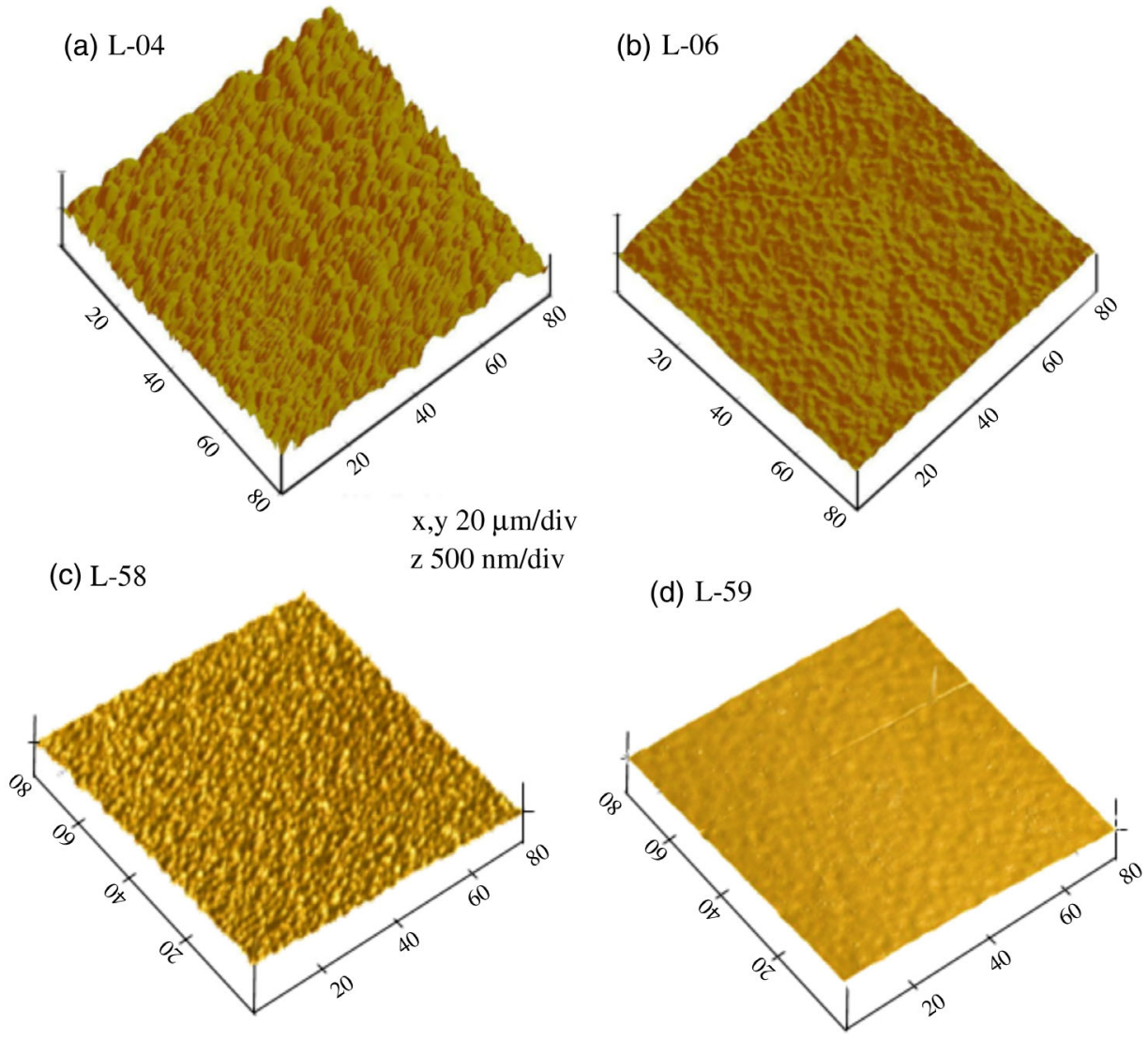

FIG. 4. AFM scan for samples (a) L-04, (b) L-06, (c) L-58 and (d) L-59 after 2N6 nitrogen doping and followed by BCP/EP. Samples L-04 and L-58 were subjected to BCP, whereas samples L-06 and L-59 were EP'ed.

over an area of $80 \times 80 \mu \mathrm{m}^{2}$ was $\sim 104 \mu \mathrm{m}$ for the BCP treated samples and $\sim 27 \mathrm{~nm}$ for EP treated fine-grain $(\mathrm{FG})$ samples. After the initial sample studies on FG Nb, two sample coupons were also cut from LG N sheet (L-58 and L-59). These LG coupons were nanopolished in the same way as the FG samples, and nitrogen doped with the same $2 \mathrm{~N} 6$ recipe. The sample coupons were subjected to $\sim 6 \mu \mathrm{m}$ $\mathrm{EP}$ and BCP respectively. AFM surface roughness measurements showed that the rms surface roughness over an area of $80 \times 80 \mu \mathrm{m}^{2}$ was $\sim 31 \mathrm{~nm}$ for the BCP treated samples and $\sim 5 \mathrm{~nm}$ for the EP treated large-grain (LG) samples.

\section{B. XPS study}

Figure 5 shows the $\mathrm{N} 1 \mathrm{~s}$ spectrum collected at $75^{\circ}$ takeoff angles for different $\mathrm{Nb}$ surfaces created in this study. The spectra of the N-doped samples (L04 and L06) show a peak 


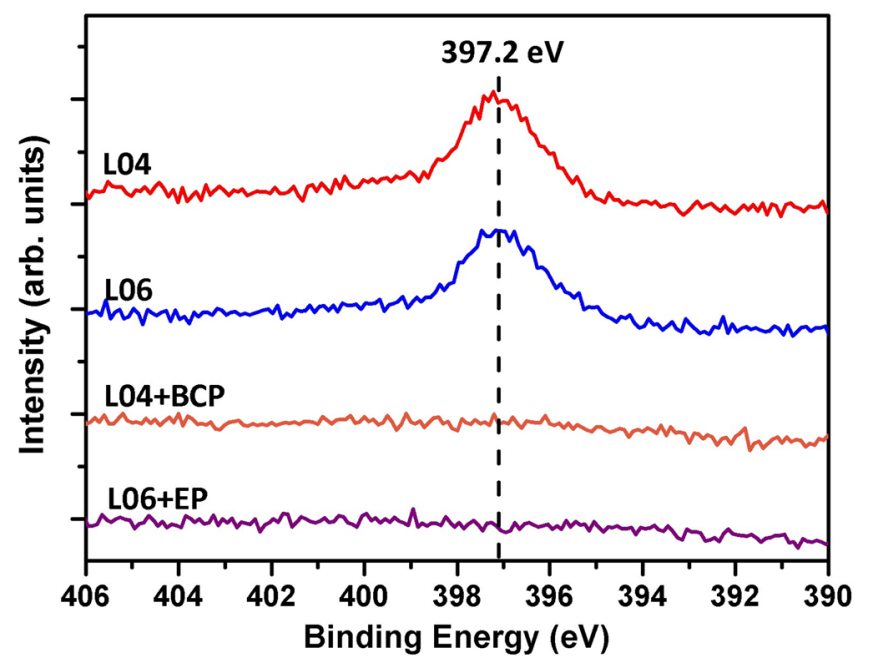

FIG. 5. N 1s XPS spectra (takeoff angle of $75^{\circ}$ ) of $\mathrm{N}$-doped $\mathrm{Nb}$ samples before and after $\sim 6 \mu \mathrm{m}$ surface removal with BCP and EP.

at B.E. $397.2 \mathrm{eV}$ which can be attributed to the niobium nitride phase. However, the spectra after $6 \mu \mathrm{m}$ surface removal with $\mathrm{BCP}$ and $\mathrm{EP}$ do not show any peak related to nitrogen or any nitrides. The suggestion based on this

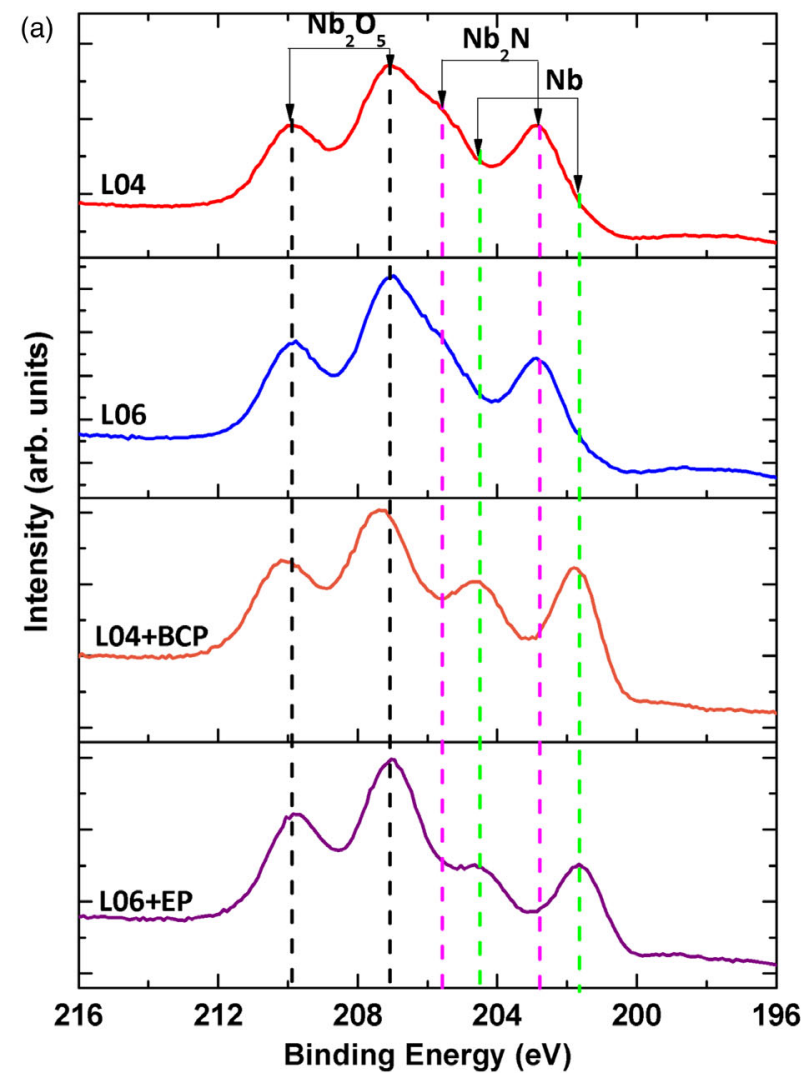

observation is that the concentration of nitrogen on the surface after $6 \mu \mathrm{m}$ surface removal is below the detection limit of XPS (0.1 at.\%).

The XPS spectra adjacent to $\mathrm{Nb} 3 \mathrm{~d}$ taken at a $75^{\circ}$ takeoff angle are shown in Fig. 6(a). All four $\mathrm{Nb}$ spectra have the dominant peak in the form of a doublet at 207.2 and $209.9 \mathrm{eV}$ which are the doublets of the $\mathrm{Nb}_{2} \mathrm{O}_{5}$ phase. In addition to the $\mathrm{Nb}_{2} \mathrm{O}_{5}$ peaks, the $\mathrm{Nb} 3 \mathrm{~d}$ spectra for the $\mathrm{N}$-doped samples (L04 and L06) also acquire a peak at B.E. $202.9 \mathrm{eV}$. The variation of this peak is consistent with the change of the peak in the $\mathrm{N} 1 \mathrm{~s}$ spectrum, so this peak could also be related to the $\mathrm{NbN}_{x}$ phase. A previous XPS study of $\beta-\mathrm{Nb}_{2} \mathrm{~N}$ films grown on the hexagonal $\mathrm{SiC}$ substrates at $850^{\circ} \mathrm{C}$ reported the appearance of the peak of the $\beta-\mathrm{Nb}_{2} N$ phase at B.E. $202.8 \mathrm{eV}$ [16]. In addition, the B.E. difference between $\mathrm{NbN}_{x}$ peaks at $\mathrm{N} 1 \mathrm{~s}$ and $\mathrm{Nb} 3 \mathrm{~d}$ spectrums in our study is $194.2 \mathrm{eV}$ which is close to the previously reported value of $194.34 \mathrm{eV}$ for $\mathrm{Nb}_{2} \mathrm{~N}$ films [17]. The result is consistent with a previously reported study where the author showed the presence of the $\mathrm{Nb}_{2} \mathrm{~N}$ phase through transmission electron microscopy [12]. The nitride phase that appears on the $\mathrm{N}$-doped $\mathrm{Nb}$ surface is $\beta-\mathrm{Nb}_{2} \mathrm{~N}$. On the $\mathrm{N}$-doped $\mathrm{Nb}$ surface, the peak from the bulk $\mathrm{Nb}$ did not exist in the $\mathrm{Nb} 3 \mathrm{~d}$ spectrum which suggests that the $\beta-\mathrm{Nb}_{2} \mathrm{~N}$ layer is significantly thick. Once the surface is chemically

(b)

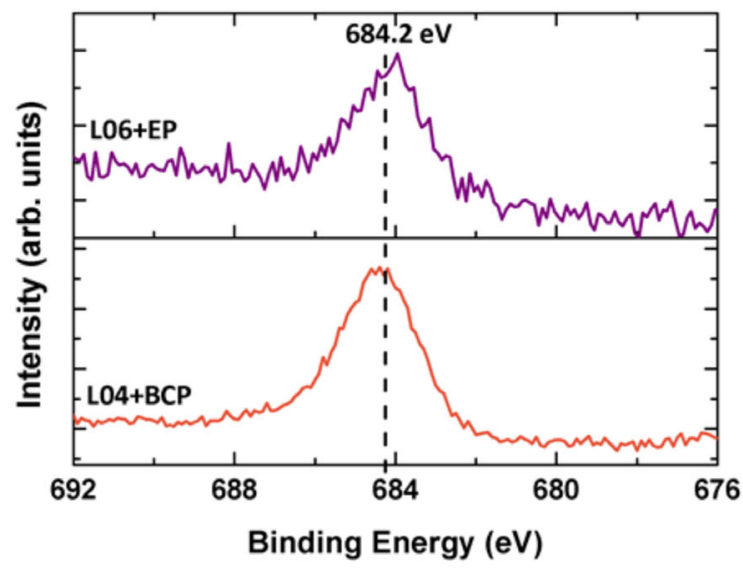

FIG. 6. (a) Nb $3 \mathrm{~d}$ XPS spectra (takeoff angle of $75^{\circ}$ ) of $\mathrm{N}$-doped $\mathrm{Nb}$ samples before and after the $\sim 6 \mu \mathrm{m}$ surface removal with BCP and EP. (b) Fluoride 1s XPS spectra (takeoff angle of $75^{\circ}$ ) at $684.2 \mathrm{eV}$ of N-doped Nb samples after the $\sim 6 \mu \mathrm{m}$ surface removal with $\mathrm{BCP}$ and EP. 

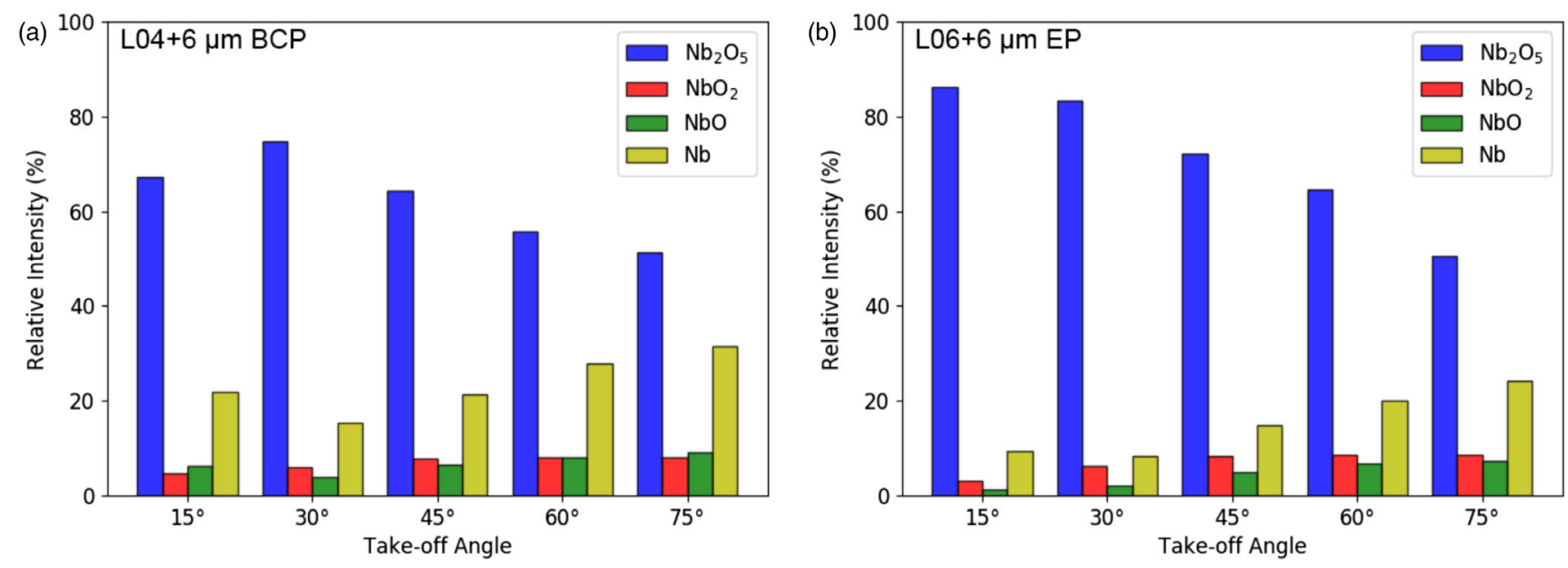

FIG. 7. Relative intensity variation of different $\mathrm{Nb}$ phases on the $\mathrm{N}$-doped $\mathrm{Nb}$ surface after $6 \mu$ m surface removal with (a) BCP and (b) EP relative to different XPS takeoff angles.

polished, removing a depth of about $6 \mu \mathrm{m}$, the $\mathrm{NbN}_{x}$ peak disappeared, and the pure $\mathrm{Nb}$ peak at a B.E. of $\sim 201.8 \mathrm{eV}$ appeared in the Nb $3 \mathrm{~d}$ XPS spectra. We also observed a peak related to fluoride in the spectra. The F 1s spectra at $75^{\circ}$ takeoff angle, shown in Fig. 6(b), shows the fluoride peak appearing at B.E. of around $684.2 \mathrm{eV}$ for both BCP and EP treated $\mathrm{Nb}$ surfaces. The concentration is, however, higher in BCP treated surface and intensity peak reduced after additional ultrasonic cleaning with deionized water. The presence of fluorine on the electropolished $\mathrm{Nb}$ surface is consistent with earlier studies [18].

Figure 7 shows the relative intensity variation of different oxides of $\mathrm{Nb}$ and the bulk $\mathrm{Nb}$ on the $\mathrm{N}$-doped $\mathrm{Nb}$ surface after $6 \mu \mathrm{m} \mathrm{BCP}$ and EP. $\mathrm{NbO}_{2}$ and $\mathrm{NbO}$ were revealed after the deconvolution of the $\mathrm{Nb} 3 \mathrm{~d}$ spectrum for lower takeoff angles where the XPS signals come from closer to the surface. The samples analyzed show lower $\mathrm{Nb}_{2} \mathrm{O}_{5}$ concentration, and higher bulk $\mathrm{Nb}$ concentration on $\mathrm{Nb}$ surfaces treated by $\mathrm{BCP}$ compared to EP. However, the higher concentration of $\mathrm{NbO}$ on the $\mathrm{BCP}$ treated sample compared to $\mathrm{EP}$. The dielectric $\mathrm{Nb}_{2} \mathrm{O}_{5}$ on the $\mathrm{BCP}$ treated $\mathrm{Nb}$ surface is thinner and less homogenous compared to the EP treated $\mathrm{Nb}$ surface. Previous XPS studies also showed lower $\mathrm{Nb}_{2} \mathrm{O}_{5}$ concentration after low-temperature baking on the $\mathrm{BCP}$ treated $\mathrm{Nb}$ surface compared to low-temperature baking on the EP treated surface and inferred the lowering of the $\mathrm{Nb}_{2} \mathrm{O}_{5}$ level to be the effect of BCP [19].

\section{TOF-SIMS results}

TOF-SIMS analyses [20] were conducted using a TOF SIMS V (ION TOF, Inc. Chestnut Ridge, NY) instrument equipped with a $\mathrm{Bi}_{n}{ }^{m+}(n=1-5, m=1,2)$ liquid metal ion gun, $\mathrm{Cs}^{+}$sputtering gun and electron flood gun for charge compensation. A higher concentration of $\mathrm{NbN}^{-}$was observed in both BCP and EP treated samples and its concentration is higher compared to the baseline non-

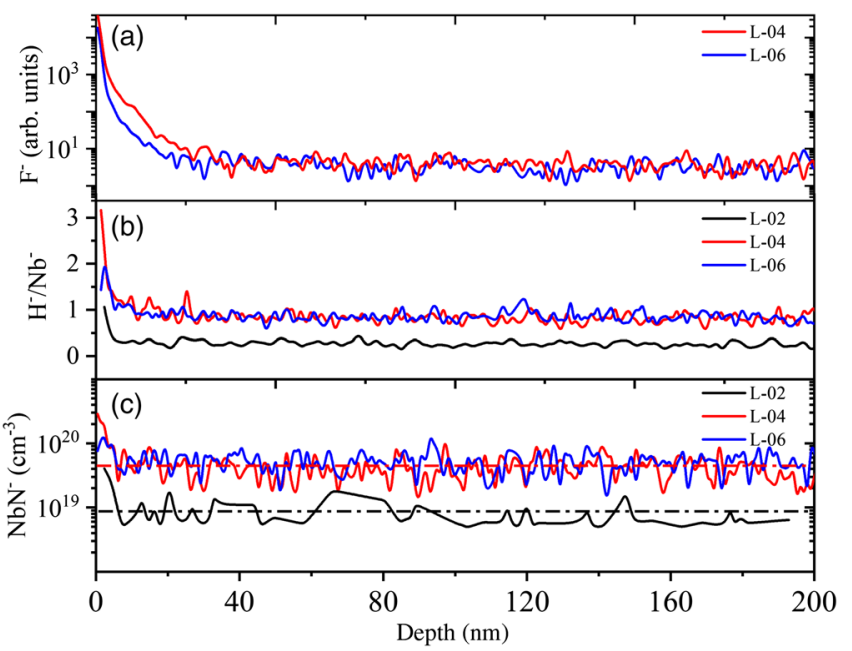

FIG. 8. TOF SIMS measurements for samples L-04 (BCP), L-06 (EP) and L-02 (nondoped); (a) concentration of $\mathrm{F}^{-}$in arbitrary unit (b) $\mathrm{H}^{-} / \mathrm{Nb}^{-}$and (b) concentration of $\mathrm{NbN}^{-}$.

nitrogen-doped samples (L-02) as shown in Fig. 8(c). Some detectable $\mathrm{F}^{-}$contamination was observed in both BCP and EP treated samples as shown in Fig. 8(a), also confirmed by XPS spectra. Furthermore, higher $\mathrm{H}^{-} / \mathrm{Nb}^{-}$ was observed on both EP and BCP treated samples compared to the sample which was subjected to only $800^{\circ} \mathrm{C} / 3 \mathrm{hrs}$ heat treatment. Hydrogen intake was typically observed during the chemical processing [21]. However, the samples cool down to $\sim 100 \mathrm{~K}$ and holding for extended periods of time did not show any hydride related features on the surface.

\section{CAVITY RF RESULTS}

The fine-grain and large-grain nitrogen-doped cavities reported on here used the same recipe and conditions as the 

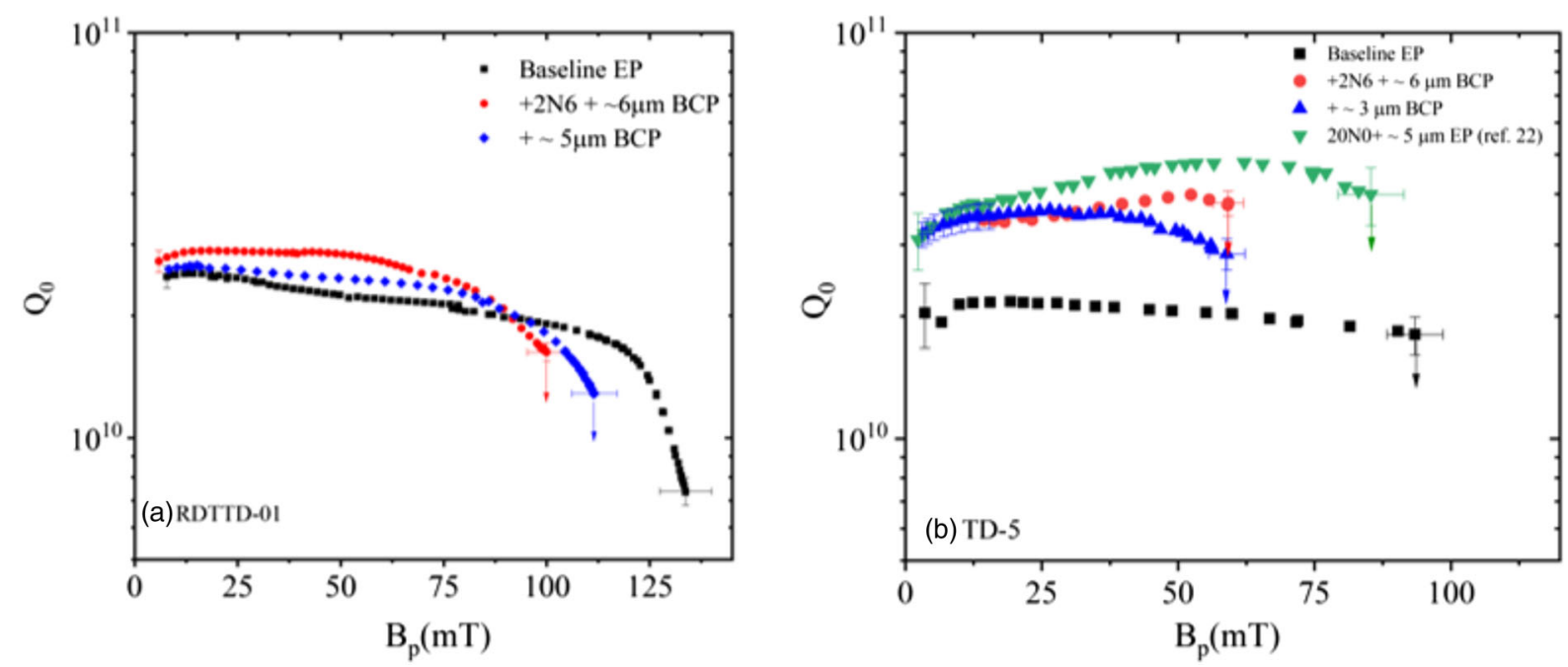

FIG. 9. $Q_{0}\left(B_{p}\right)$ for (a) RDTTD-01 and (b)TD-5 after nitrogen doping followed BCP. For reference the data also are shown for TD-05 after EP from Ref. [22]. The arrows indicate the limitation by quench.

coupon samples but only used BCP for the final surface removal. Standard procedures were followed to clean the cavity surface in preparation for an rf test: degreasing in ultrapure water with a detergent and ultrasonic agitation, high pressure rinsing with ultrapure water, drying in the ISO4/5 cleanroom, assembly of flanges with rf feedthroughs and pump out ports and evacuation. The cavity was inserted in a vertical cryostat and cooled to $4.5 \mathrm{~K}$ with liquid helium using the standard Jefferson Lab cooldown procedure in a residual magnetic field of $<2 \mathrm{mG}$. This procedure results in a temperature difference between the two irises $\Delta T>4 \mathrm{~K}$ when the equator temperature crosses the superconducting transition temperature $(\sim 9.2 \mathrm{~K})$ to ensure the good flux expulsion conditions. The cavity immersed in the helium bath of temperature $2.0 \mathrm{~K}$ is excited using a phase-locked loop to measure the quality factor as a function of the peak magnetic field on the inner cavity surface.

Figure 9 shows the $Q\left(B_{p}\right)$ curve for the two cavities measured at $2.0 \mathrm{~K}$. As shown in Fig. 9(a) the cavity RDTTD-01 showed an overall increase in $Q_{0}$ at low and medium field range $(<90 \mathrm{mT})$ with $\sim 24 \%$ increase at $B_{p}=$ $67 \mathrm{mT}\left(E_{\text {acc }}=16 \mathrm{MV} / \mathrm{m}\right)$ in $Q_{0}$ after $\sim 6 \mu \mathrm{m}$ surface removal by BCP with quench field of $\sim 100 \mathrm{mT}$. The quenched field was improved to $\sim 110 \mathrm{mT}$ with an additional $\sim 5 \mu \mathrm{m}$ BCP but with lower $Q_{0}$. Figure 9(b) showed an increase in $Q_{0}$ with $Q$-rise phenomenon and before the cavity was limited by quenching at $B_{p} \sim 60 \mathrm{mT}$ $\left(E_{\text {acc }} \sim 14 \mathrm{MV} / \mathrm{m}\right)$ after $\sim 6 \mu \mathrm{m}$ inner surface removal using BCP. With an additional $\sim 3 \mu \mathrm{m} \mathrm{BCP,} \mathrm{the} \mathrm{quenched}$ field did not change, however the $Q_{0}\left(B_{p}\right)$ curve begins to show a decrease in $Q_{0}$ above $B_{p} \sim 40 \mathrm{mT}$. Also shown is the previously published data from the same cavity with nitrogen doping but the final surface removal being $\sim 5 \mu \mathrm{m}$ EP [22]. It is to be noted that the baseline measurement on cavity RDTTD-01 was limited by high field $Q$-slope.

\section{DISCUSSION}

This study indicates that the surface compositions are similar in coupon samples after $\mathrm{N}$ doping and subsequent BCP or EP. In two different polycrystalline samples nonsuperconducting $\mathrm{Nb}_{2} \mathrm{~N}$ are formed as shown in Figs. 1 and 5 after $\mathrm{N}$ doping. Postprocessing leading to $\sim 6 \mu \mathrm{m}$ surface polishing by $\mathrm{BCP}$ or EP leads to complete removal of $\mathrm{Nb}_{2} \mathrm{~N}$ phases as shown in Fig. 6(a). There are no significant surface composition variations between $\mathrm{BCP}$ and EP removal as indicated by XPS, and SIMS.

Coupon samples indicate that the major variations between BCP and EP are the surface roughness as indicated qualitatively by secondary electron imaging (Fig. 2) and quantitatively by AFM measurements (Fig. 4). EP produces much smoother surfaces than BCP, which could be due to removal rates in grain boundaries and grains with different orientations produced in $\mathrm{BCP}$ using the traditional recipes. It is still unclear if reducing temperature, etching times, or balancing the $\mathrm{HF}: \mathrm{HNO}_{3}$ ratios could produce a more uniform surface removal. Also, $\mathrm{F}^{-}$concentration is slightly higher in the BCP processed samples than EP samples. This could be a consequence of higher surface roughness in $\mathrm{BCP}$ which could lead to several convex regions on the material surface where fluoride salts can reside even after extensive cleaning. It is likely that higher-pressure rinse like a HPR could reduce the $\mathrm{F}^{-}$concentration. The $\mathrm{NbN}^{-}$concentration measured on replica coupon samples is around $10^{19}-10^{20} / \mathrm{cm}^{3}$ as indicated by the SIMS in Fig. 8(c). If $\mathrm{NbN}^{-}$concentration alone is the determining factor in maintaining high $\mathrm{Q}$ behavior in the midfield range, the $20 \mathrm{~N} 0$ nitrogen doping protocol followed by $40 \mu \mathrm{m}$ removal shows the same level of $\mathrm{N}$ concentration range of $10^{19}-10^{20} / \mathrm{cm}^{3}$ [22], as observed after a $2 \mathrm{~N} 6$ recipe and $10 \mu \mathrm{m}$ removal [23]. Cavity rf testing has typically shown that the EP-treated cavities typically achieved a higher 
accelerating gradient compared to flash BCP-treated cavities [24]. The combination of nitrogen doping and optimal electropolishing resulted in higher quality factor increasing with the accelerating gradient before it quenched at lower gradient compared to baseline $\mathrm{EP} / \mathrm{BCP}$ cavities. The increase in quality factor has been explained by the decrease in BCS surface resistance and the $Q$-rise phenomenon mainly arises from the broadening of the peak of density of states by the rf currents in the $\mathrm{Nb}$ superconductor $[25,26]$.

The coupon characterization showed no distinct difference between the EP and BCP treated surfaces. The only noticeable change being the surface roughness as seen from high magnification SEM and AFM measurements. In the past the $Q$-slope in the medium field was linked to the surface roughness via the local magnetic field enhancement and $Q$-slope starts at lower field for $\mathrm{BCP}$ treated cavities compared to EP treated FG cavities [27-29]. BCP treated niobium has a typical roughness that is about an order of magnitude higher than that for EP treated samples [30]. However, it can vary with the starting surfaces, acid mixtures and temperatures. The rms roughness we observed on nanopolished and N-doped surfaces followed by $\mathrm{BCP}$ and $\mathrm{EP}$ surface removal is comparable to the previous studies [31,32]. A much smoother surface was obtained on LG Nb consistent with previous results [33].

One of the distinct features we observed based on cavity rf measurement on BCP treated fine-grain cavity is the lack of a pronounced $Q$-rise phenomenon, which is contrary to those observed in EP treated cavities, even though there is no significant surface composition as seen from sample studies. Sample coupon studies suggest that the topography may play a role in determining the $Q\left(B_{p}\right)$ curve, contrary to the widely accepted explanation of $Q$-rise phenomenon as a result of reduction in BCS surface resistance due to the presence of impurities ( $\mathrm{Ti}$ or $\mathrm{N}$ ) within the rf penetration depth $[24,25]$. The case is different for LG niobium as both BCP and EP resulted in the $Q$-rise phenomenon at low field and the $Q$-slope appeared with additional BCP. It should be noted that the differences in removal rate with grain orientation and at grain boundaries are significantly reduced due to the lower density of grain boundaries in LG Nb. Single grains could have smoother surfaces depending on the orientation due to BCP. In a recent systematic sample coupon study on nitrogen-doped FG samples, a variation in the precipitation of normal conducting $\mathrm{Nb}_{2} N$ with grain orientation was observed, and it was suggested that this may be due to grain-grain differences in nitrogen accumulation and transport [34].

\section{CONCLUSION}

The coupon study of nitrogen-doped high purity finegrain niobium showed smoother surfaces for the EP samples compared to the BCP coupons as is typically observed even in the past on non-nitrogen-doped SRF surfaces. The near surface elemental concentrations based on the XPS and TOF-SIMS measurements did not show any significant difference as a result of chemical treatment. Radio-frequency testing of the cavity made from fine-grain $\mathrm{Nb}$ showed a moderate increase in quality factor in medium field range $(<20 \mathrm{MV} / \mathrm{m})$ over the baseline EP surface treatment. The large-grain cavity showed a higher increase in $Q_{0}$ compared to the fine-grain niobium with the same amount of material removal via $\mathrm{BCP}$, comparable to fine-grain EP cavities [11]. The enhancement in $Q_{0}$ in the EP treated (20N0) cavity is much higher than for the BCP treated (2N6) FG cavities, which is about $40 \%$ on average at $B_{p} \sim 67 \mathrm{mT}\left(E_{\text {acc }}=16 \mathrm{MV} / \mathrm{m}\right)$ based on recent LCLS-II cavities production [11]. BCP may be a particularly attractive postprocessing option for after doping of complex shaped cavities where the EP process is harder to achieve. The origins of the superiority of the EP over the BCP on these nitrogen-doped cavities still need further investigation.

\section{ACKNOWLEDGMENTS}

We would like to acknowledge Jefferson Lab technical staff for the cavity surface processing and cryogenic support and Elaine Zhou at Analytical Instrumentation Facility (AIF), North Carolina State University, for SIMS measurements. We would like to acknowledge Olga Trifimova for her help with AFM. The work done at Florida State University is supported by the U.S. Department of Energy, Office of Science, Office of High Energy Physics under Award No. DE-SC 0009960 (FSU) and the State of Florida. Additional support for the National High Magnetic Field Laboratory facilities is from the National Science Foundation Cooperative Agreements No. DMR-1157490 and No. DMR-1644779. This manuscript has been authored by Jefferson Science Associates, LLC under U.S. DOE Contract No. DE-AC0506OR23177.

[1] P. Dhakal, G. Ciovati, and G. R. Myneni, A path to higher Q0 in large grain niobium cavities, in Proceedings of the 3rd International Particle Accelerator Conference, New Orleans, LA, 2012, edited by Jacow Collaboration (IEEE, Piscataway, NJ, 2012), p. 2426 [arXiv:1205.6736].

[2] P. Dhakal et al., Effect of high temperature heat treatments on the quality factor of a large-grain superconducting radio-frequency niobium cavity, Phys. Rev. ST Accel. Beams 16, 042001 (2013).

[3] P. Dhakal, G. Ciovati, P. Kneisel, and G. R. Myneni, Enhancement in quality factor of SRF niobium cavities by material diffusion, IEEE Trans. Appl. Supercond. 25, 1 (2015).

[4] A. Grassellino, A. Romanenko, D. Sergatskov, O. Melnychuk, Y. Trenikhina, A. Crawford, A. Rowe, M. Wong, T. Khabiboulline, and F. Barkov, Nitrogen and 
argon doping of niobium for superconducting radio frequency cavities: A pathway to highly efficient accelerating structures, Supercond. Sci. Technol. 26, 102001 (2013).

[5] A. Grassellino et al., Unprecedented quality factors at accelerating gradients up to $45 \mathrm{MV} \mathrm{m}^{-1}$ in niobium superconducting resonators via low temperature nitrogen infusion, Supercond. Sci. Technol. 30, 094004 (2017).

[6] P. N. Koufalis, F. Furuta, M. Ge, D. Gonnella, J. J. Kaufman, M. Liepe, J. T. Maniscalco, and R. D. Porter, Low temperature nitrogen baking of $Q_{0}$ SRF cavities, in Proceedings of LINAC2016, East Lansing, MI, edited by Jacow Collaboration (JACoW, Geneva, Switzerland, 2016), p. 472, https://doi.org/10.18429/JACoW-LINAC2016-TUPRC025.

[7] P. Dhakal, S. Chetri, S. Balachandran, P. J. Lee, and G. Ciovati, Effect of low temperature baking in nitrogen on the performance of a niobium superconducting radio frequency cavity, Phys. Rev. Accel. Beams 21, 032001 (2018).

[8] H. Tian, Ph.D. thesis, The College of William and Mary, 2008.

[9] Z. H. Sung, A. Dzyuba, P. J. Lee, D. C. Larbalestier, and L. D. Cooley, Evidence of incomplete annealing at $800{ }^{\circ} \mathrm{C}$ and the effects of $120^{\circ} \mathrm{C}$ baking on the crystal orientation and the surface superconducting properties of cold-worked and chemically polished Nb, Supercond. Sci. Technol. 28, 075003 (2015).

[10] B. Aune et al., Superconducting TESLA cavities, Phys. Rev. ST Accel. Beams 3, 092001 (2000).

[11] D. Gonnella et al., Industrialization of the nitrogen-doping preparation for SRF cavities for LCLS-II, Nucl. Instrum. Methods Phys. Res., Sect. A 883, 143 (2018).

[12] Y. Trenikhina, A. Grassellino, O. S. Melnychuk, and A. Romanenko, Characterization of nitrogen doping recipes for the Nb SRF cavities, in Proceedings of the SRF2015, Whistler, BC, Canada, edited by Jacow Collaboration, https://www.jacow.org.

[13] K. Saito et al., Superiority of electropolishing over chemical polishing on high gradients, Part. Accel. 60, 193 (1998).

[14] F. Barkov, A. Romanenko, and A. Grassellino, Direct observation of hydrides formation in cavity-grade niobium, Phys. Rev. ST Accel. Beams 15, 122001 (2012).

[15] P. Grag, S. Balachandran, I. Adlakha, P. J. Lee, T. R. Bieler, and K. N. Solanki, Revealing the role of nitrogen on hydride nucleation and stability in pure niobium using first principles calculations, Supercond. Sci. Technol. 31, 115007 (2018).

[16] D. S. Katzer, N. Nepal, D. J. Meyer, B. P. Downey, V. D. Wheeler, D. F. Storm, and M. T. Hardy, Metallic $\beta-\mathrm{Nb}_{2} N$ films epitaxially grown by $\mathrm{MBE}$ on hexagonal $\mathrm{SiC}$ substrates, MRS Adv. 1, 127 (2016).

[17] R. Sanjinés, M. Benkahoul, M. Papagno, and F. Levy, Electronic structure of $\mathrm{Nb}_{2} \mathrm{~N}$ and $\mathrm{NbN}$ thin films, J. Appl. Phys. 99, 044911 (2006).

[18] J. Jouve, Y. Belkacem, and C. Severac, X-ray photoelectron spectroscopy study of phosphorus incorporation in anodic oxide films on niobium, Thin Solid Films 139, 67 (1986).
[19] K. Kowalski, A. Bernasik, W. Singer, X. Singer, and J. Camra, In situ XPS investigation of the baking effect on the surface oxide structure formed on niobium sheets used for superconducting rf cavity production, in Proceedings of SRF'03, Lubeck/Travemunde, Germany, edited by Jacow Collaboration (2003), http://www.jacow .org, p. 610.

[20] A. M. Belu, D. J. Graham, and D. G. Castner, Time-offlight secondary ion mass spectrometry: Techniques and applications for the characterization of biomaterial surfaces, Biomaterials 24, 3635 (2003).

[21] R.E. Ricker and G. R Myneni, Evaluation of the propensity of niobium to absorb hydrogen during fabrication of superconducting radio frequency cavities for particle accelerators, J. Res. Natl. Inst. Stand. Technol. 115, 353 (2010).

[22] A. D. Palczewski, P. Dhakal, and C. E. Reece, Analysis of BCS rf loss dependence on N-doping protocols, in Proceedings of SRF 15, Whistler, BC, Canada, edited by Jacow Collaboration, http://www .jacow.org.

[23] D. Gonnella, M. Ge, F. Furura, and M. Liepe, Nitrogen treated cavity testing at Cornell, in Proceedings of LINAC 14, Geneva, Switzerland, edited by Jacow Collaboration, http://www.jacow.org.

[24] W. Singer et al., Production of superconducting 1.3-GHz cavities for the European x-ray free electron laser, Phys. Rev. Accel. Beams 19, 092001 (2016).

[25] A. Gurevich, Reduction of Dissipative Nonlinear Conductivity of Superconductors by Static and Microwave Magnetic Fields, Phys. Rev. Lett. 113, 087001 (2014).

[26] G. Ciovati, P. Dhakal, and A. Gurevich, Decrease of the surface resistance in superconducting niobium resonator cavities by the microwave field, Appl. Phys. Lett. 104, 092601 (2014).

[27] J. Knobloch, R. L. Geng, M. Liepe, and H. Padamsee, in Proceedings of the 9th Workshop on RF Superconductivity Los Alamos National Laboratory, Santa Fe, NM, edited by Jacow Collaboration, http://www.jacow.org.

[28] C. Xu, C.E. Reece, and M. J. Kelly, Simulation of nonlinear superconducting rf losses derived from characteristic topography of etched and electropolished niobium surfaces, Phys. Rev. Accel. Beams 19, 033501 (2016).

[29] T. Kubo, Magnetic field enhancement at a pit on the surface of a superconducting accelerating cavity, Prog. Theor. Exp. Phys. (2015) 073G01 (2015).

[30] C. Xu, H. Tian, C. E. Reece, and M. J. Kelly, Enhanced characterization of niobium surface topography, Phys. Rev. ST Accel. Beams 14, 123501 (2011).

[31] H. Tian, C. E. Reece, M. J. Kelley, S. Wang, L. Plucinski, K. E. Smith, and M. M. Nowell, Surface studies of niobium chemically polished under conditions for superconducting radio frequency (SRF) cavity production, Appl. Surf. Sci. 253, 1236 (2006).

[32] H. Tian and C.E. Reece, Evaluation of the diffusion coefficient of fluorine during the electropolishing of niobium, Phys. Rev. ST Accel. Beams 13, 083502 (2010). 
[33] P. Kneisel, G. Ciovati, P. Dhakal, K. Saito, W. Singer, X. Singer, and G. R. Myneni, Review of ingot niobium as a material for superconducting radio-frequency accelerating cavities, Nucl. Instrum. Methods Phys. Res., Sect. A 774, 133 (2015).
[34] J. K. Spradlin, A. D. Palczewski, H. Tian, and C. E. Reece, Analysis of surface nitrides created during doping heat treatments of niobium, in Proceedings of the SRF 19 Dresden Germany, edited by Jacow Collaboration, http:// www.jacow.org. 